\title{
ESTRUTURA FITOSSOCIOLÓGICA DE UM FRAGMENTO NATURAL DE FLORESTA INUNDÁVEL EM ÁREA DE ORIZICULTURA IRRIGADA, MUNICÍPIO DE LAGOA DA CONFUSÃO, TOCANTINS ${ }^{1}$
}

\author{
Elizabeth Rodrigues Brito², Sebastião Venâncio Martins ${ }^{3}$, Ary Teixeira de Oliveira Filho ${ }^{4}$, Elias Silva ${ }^{3}$ e
} Alexandre Francisco da Silva ${ }^{5}$

\begin{abstract}
RESUMO - Os fragmentos naturais de florestas inundáveis conhecidos como ipucas localizam-se na planície do Araguaia, sob a forma de depressões naturais, que no Estado do Tocantins estão situados em áreas de planícies de inundação, que favorece seu alagamento e, conseqüentemente, o maior tempo de retenção da água em épocas de elevada precipitação. O presente estudo foi desenvolvido na fazenda Lago Verde, Município de Lagoa da Confusão, Tocantins, situado entre as coordenadas UTM: 643586 e 644060 leste e 8792795 e 8799167 norte. O objetivo do trabalho foi caracterizar a estrutura de um fragmento de floresta inundável de aproximadamente um hectare, inserido em área de orizicultura irrigada. Para o levantamento fitossociológico, foram amostrados todos os indivíduos arbustivo-arbóreos com perímetro a $1,30 \mathrm{~m}$ do solo (PAP) $\geq 15 \mathrm{~cm}$. Ao todo, foram encontrados 807 indivíduos, 35 famílias e 70 espécies. As espécies com maior VC, em ordem decrescente, foram Hirtella racemosa Lam., Qualea multiflora Mart. e Cecropia pachystachya Trécul. As famílias mais ricas em espécies foram Fabaceae (9), Vochysiaceae (6), Annonaceae e Malvaceae (4). O índice de diversidade de Shannon (H') foi de 3,44. A distribuição de classes de diâmetro apresentou curva na forma de "J" invertido, estando a maioria dos indivíduos na primeira classe.
\end{abstract}

Palavras-chave: Florestas inundáveis, fitossociologia, classes de diâmetro e ipucas.

\section{PHYTOSOCIOLOGICAL STRUCTURE OF A NATURAL FRAGMENT OF FLOODPLAIN FOREST IN AREA OF IRRIGATED RICE CULTIVATION, MUNICIPAL DISTRICT OF LAGOA DA CONFUSÃO, TOCANTINS, BRAZIL}

\begin{abstract}
The natural fragments of floodplain forests known as "ipucas" are located in the Araguaia plain, in form of natural depressions, which are located in areas of floodplains in the State of Tocantins, favoring its flooding and, consequently, the largest period of water retention during periods of high precipitation. The present study was developed at Lagoa Verde farm, municipal district of Lagoa da Confusão, Tocantins between the coordinates UTM: 643586 and 644060 east and 8792795 and 8799167 north. The objective of the work was to characterize the structure of a floodplain forest fragment of approximately one-hectare area, inserted in area of irrigated rice cultivation. For the phytosociological survey, all the tree/shrub individuals with perimeter at $1.30 \mathrm{~m}(\mathrm{PBH}) \geq 15 \mathrm{~cm}$ were sampled. On the whole, 807 individuals, 35 families and 70 species were sampled. The species with higher VC, in descending order, were Hirtella racemosa Lam., Qualea
\end{abstract}

\footnotetext{
${ }^{1}$ Recebido em 21.10.2005 e aceito para publicação em 09.08.2004.

${ }^{2}$ Instituto Natureza do Tocantins - Naturatins, Coordenadoria de Ordenamento Florestal, AANE 40, QI 02, Lt. 3A, Alameda 1,77054-080,Palmas-TO.E-mail: <erbrito@vicosa.ufv.br.>.

${ }^{3}$ Departamento de Engenharia Florestal da Universidade Federal de Viçosa, 36571-000 Viçosa-MG. E-mail:<venancio@ufv.br>.

${ }^{4}$ Departamento de Ciências Florestais da Universidade Federal de Lavras, 37200-000 Lavras-MG.

${ }^{5}$ Departamento de Biologia Vegetal da Universidade Federal de Viçosa, 36571-000 Viçosa-MG.
} 
multiflora Mart. and Cecropia pachystachya Trécul. The richest families in species were Fabaceae (9), Vochysiaceae (6), Annonaceae and Malvaceae (4). The Shannon diversity index ( $H^{\prime}$ ') was 3.44. The distribution of diameter classes showed an inverted " $J$ " curve, with most of the individuals being in the first class.

Keywords: Floodplain forests, phytosociology, diameter classes, ipucas.

\section{INTRODUÇÃO}

O Brasil é conhecido como um país megadiverso, por possuir um patrimônio genético incomparável nos seus mais ricos ecossistemas (FORERO, 1989; MITTERMEIR et al., 1992; FACHIM e GUARIM, 1995; BORÉM e OLIVEIRA-FILHO, 2002). Considerado o segundo maior bioma do país, o Cerrado ocorre em todo o Brasil central, em partes da Amazônia, Nordeste, Sudeste e em pequenas "ilhas" no Paraná (FELFILI e SILVAJÚNIOR, 1988; EITEN, 1994; OLIVEIRA-FILHO e RATTER, 1995; RATTER et al., 1997). Abrange cerca de dois milhões de $\mathrm{km}^{2}$, sendo superado apenas pela vegetação amazônica, que possui aproximadamente 3,5 milhões de km² (RATTER et al., 1997). Entretanto, grande parte das áreas de Cerrado já não possui mais a cobertura vegetal original, sendo atualmente ocupada por paisagens antrópicas (COUTINHO, 1990; FELFILI et al., 1993; RATTER et al., 1997; FELFILI et al., 2002; SILVA et al., 2002; FIEDLER et al., 2004). Além disso, a supressão da vegetação condiciona à fragmentação que se avizinha a usos agrícolas e a outras formas de uso (SAUNDERS et al., 1991).

Com a criação do Estado de Tocantins em 1989, houve pronunciado aumento da expansão da fronteira agropecuária nas áreas de Cerrado. Na planície do Araguaia, a sudoeste do Estado, a orizicultura irrigada expandiu-se por ser essa uma área plana e de fácil mecanização. Nessa região predomina uma área de transição do Cerrado com a Floresta Amazônica e que periodicamente sofre inundação na estação chuvosa durante quatro a cinco meses, abrangendo aproximadamente $600.000 \mathrm{~km}^{2}$ de extensão, sendo que a maior representatividade de área inundável se localiza na Ilha do Bananal. A região apresenta todas as fisionomias do bioma Cerrado, que inclui o Campo Limpo, o Campo Sujo, o Campo Cerrado, o Cerrado stricto sensu e o Cerradão, que são predominantes na paisagem mais as inclusões florestais, como as florestas de galeria e também um tipo vegetacional peculiar de fragmentos naturais de floresta inundável, denominado regionalmente de "ipucas" ou "impucas" (EITEN, 1985).
Os fragmentos de florestas inundáveis na planície no Estado do Tocantins restringem-se às áreas de planícies de inundação da bacia do rio Araguaia em situações bem definidas, sob a forma de depressões naturais que favorecem seu alagamento e, conseqüentemente, o maior tempo de retenção da água em épocas de maior precipitação; somada a essa condição ocorre naturalmente em forma de fragmentos isolados, apresentando peculiaridades florísticas, estruturais e fisionômicas dos demais tipos vegetacionais do entorno.

$\mathrm{Na}$ área em estudo, os fragmentos de florestas inundáveis surgem dentro das subformações da fisionomia campestre (campo sujo e, ou campo limpo) regionalmente denominados varjões, que Eiten (1985) chamou de um tipo de pantanal caracterizado como um terreno não-florestado, anualmente encharcado ou rasamente inundado por quatro a cinco meses, entre dezembro e abril, e fortemente ressecado durante a estação seca (junho a setembro) e em vários locais com a presença de campos murundus.

Estudos florísticos e fitossociológicos no Estado do Tocantins são escassos, e o conhecimento fitossociológico das florestas inundáveis no Município de Lagoa da Confusão, a sudoeste do Estado, inseridas em área antropizada, torna-se de fundamental importância, por serem áreas singulares no contexto do bioma Cerrado. Dessa forma, os objetivos deste trabalho foram caracterizar e analisar a estrutura fitossociológica da formação vegetal denominada floresta inundável e fornecer dados que possam contribuir para o conhecimento e preservação desses fragmentos naturais que estão sofrendo fortes pressões antrópicas no Estado do Tocantins.

\section{MATERIAL E MÉTODOS}

\section{1. Área de estudo}

Este estudo foi realizado em um fragmento natural de floresta inundável no Município de Lagoa da Confusão, Tocantins, numa propriedade rural denominada fazenda Lago Verde, situada entre as coordenadas UTM: 643586 e 655060 leste e 8792795 
a 8799167 norte (MARTINS et al., 2002), nas proximidades de duas Unidades de Conservação: Parque Nacional do Araguaia e Parque Indígena do Araguaia, no Estado do Tocantins. A altitude está em torno de $180 \mathrm{~m}$, e a temperatura média anual apresenta-se em torno de 24 ${ }^{\circ} \mathrm{C}$. O total médio anual de precipitação é de $1.700 \mathrm{~mm}$, entre outubro e abril. De acordo com o sistema de Thornthwaite-Mather, o clima da região é do tipo B2rA'a' - clima úmido com pequena ou nula deficiência hídrica. Os solos da região apresentam manchas de Gleissolos e Plintossolos (EMBRAPA, 1999); são solos pouco profundos e estão sob forte influência do lençol freático (SEPLAN/TO, 2002). Na região, encontram-se fisionomias de Cerrado, florestas de galerias e os fragmentos naturais de florestas inundáveis. Essa região passa, desde os finais dos anos 1960 e principalmente nos anos 1970 e 80, por um intenso processo de ocupação, favorecido pela topografia plana propícia à mecanização agrícola e à implantação da pecuária.

\subsection{Amostragem fitossociológica}

Realizou-se o método de censo, ou seja, a marcação numérica e identificação taxonômica de todos os indivíduos arbustivo-arbóreos com circunferência a 1,30 m do solo $(\mathrm{CAP}) \geq 15 \mathrm{~cm}$, em um fragmento de aproximadamente 1 ha, inserido em área de orizicultura irrigada. Foi coletado material botânico dos indivíduos amostrados para identificação, a qual foi realizada por comparação com material dos herbários da Universidade Federal de Viçosa, Universidade de Brasília e Universidade Federal de Lavras. Os nomes das espécies e respectivas autoridades foram confirmados e atualizados por bibliografia específica e também através do site do Missouri Botanical Garden (http://www.mobot.org/w3t/search/vast.html). O sistema de classificação adotado foi o APG II (2003).

Os parâmetros fitossociológicos calculados foram: densidade relativa, dominância relativa e, a partir desses, o valor de cobertura (MUELLER-DOMBOIS e ELLENBERG, 1974). Foram calculados o índice de diversidade de Shannon (H') e a equabilidade (J') (PIELOU, 1975) como indicadores de heterogeneidade. Para calcular os referidos parâmetros, foi utilizado o programa Fitopac 1 (SHEPHERD, 1996).

A distribuição de freqüência diamétrica foi feita mediante o computo dos indivíduos amostrados de cada uma das espécies dentro da classe diamétrica a que pertencem (HARPER, 1977). As classes de diâmetro foram estabelecidas com amplitude de $5 \mathrm{~cm}$, a partir do diâmetro mínimo de inclusão.

\subsection{Caracterização do solo}

Para a caracterização do solo foi coletada uma amostra composta, obtida a partir de 10 amostras simples coletadas de forma aleatória, na profundidade de 0 a $20 \mathrm{~cm}$ no fragmento. A amostra foi submetida a análises química e granulométrica no Laboratório de Solos da Universidade Federal de Viçosa, seguindo-se metodologia da Embrapa (1979).

\section{RESULTADOS E DISCUSSÃO}

\subsection{Análise do solo}

Os resultados das análises química e granulométrica estão apresentados no Quadro 1.

Verifica-se que o solo do fragmento é distrófico, com alto teor de alumínio e baixa saturação de bases. A elevada acidez e a baixa fertilidade química do solo do fragmento condiz com o contexto pedológico em que está inserido, ou seja, o ambiente de Cerrado. O resultado da análise textural indicou que o solo do fragmento se caracteriza como argiloso.

Quadro 1 - Análises químicas e granulométricas de solo no fragmento de floresta inundável no município de Lagoa da Confusão, Tocantins (SB: soma de bases trocáveis; t: capacidade de troca catiônica efetiva; T: capacidade de troca catiônica em pH 7; V: saturação por bases; m: saturação por alumínio)

Table 1 -Soil chemical and granular analyses in the flooded forest fragment in the municipal district of Lagoa da Confusão, Tocantins. (SB: sum of exchangeable bases; $t$ : effective cation exchange capacity; $T$ : cation exchange capacity in $\mathrm{pH} 7$; V: base saturation; $m$ : Aluminum saturation)

\begin{tabular}{|c|c|}
\hline Parâmetro & Resultado \\
\hline$P\left(\mathrm{mg} . \mathrm{dm}^{3}\right)$ & 0,7 \\
\hline P-remanescente (mg.l) & 11,1 \\
\hline $\mathrm{K}^{+}\left(\mathrm{mg} \cdot \mathrm{dm}^{-3}\right)$ & 73 \\
\hline $\mathrm{Ca}^{++}\left(\mathrm{cmol}_{\mathrm{c}} \cdot \mathrm{dm}^{-3}\right)$ & 0,97 \\
\hline $\mathrm{Mg}^{++}\left(\mathrm{cmol}_{\mathrm{c} .} \mathrm{dm}^{-3}\right)$ & 1,33 \\
\hline $\mathrm{Al}^{+++}\left(\mathrm{cmol}_{\mathrm{c}} \mathrm{dm}^{-3}\right)$ & 0,60 \\
\hline $\mathrm{H}^{+}+\mathrm{Al}^{+++}\left(\mathrm{cmol}_{\mathrm{c} .} \mathrm{dm}^{-3}\right)$ & 9,1 \\
\hline $\mathrm{SB}-$ soma de bases $\left(\mathrm{cmol}_{\mathrm{c}} \cdot \mathrm{dm}^{-3}\right)$ & 2,49 \\
\hline $\mathrm{t}\left(\mathrm{cmol}_{\mathrm{c} .} \mathrm{dm}^{-3}\right)$ & 3,09 \\
\hline $\mathrm{T}\left(\mathrm{cmol}_{\mathrm{c}} \cdot \mathrm{dm}^{-3}\right)$ & 11,59 \\
\hline $\mathrm{V}(\%)$ & 21,05 \\
\hline $\mathrm{m}(\%)$ & 19,4 \\
\hline pH (água) & 5,59 \\
\hline Areia grossa $\left(\mathrm{dag} \cdot \mathrm{kg}^{-1}\right)$ & 13 \\
\hline Areia fina $\left(\mathrm{dag} \cdot \mathrm{kg}^{-1}\right)$ & 14 \\
\hline Silte $\left(\right.$ dag. $\left.\mathrm{kg}^{-1}\right)$ & 21 \\
\hline $\operatorname{Argila}\left(\mathrm{dag} \cdot \mathrm{kg}^{-1}\right)$ & 52 \\
\hline Classe textural & Argila \\
\hline
\end{tabular}




\subsection{Fitossociologia}

Foram identificados 807 indivíduos, 35 famílias e 70 espécies. Os indivíduos mortos em pé somaram 26. A densidade absoluta estimada foi de 707,9 indivíduos por hectare, área basal de $12,92 \mathrm{~m}^{2} /$ ha, diâmetro médio individual de $12,99 \mathrm{~cm}$, altura média de $7,52 \mathrm{~m}$ e o volume de $0,181 \mathrm{~m}^{3} / \mathrm{ha}$. As famílias que mais contribuíram para a riqueza florística foram: Fabaceae com nove espécies $(12,85 \%)$, Vochysiaceae com seis espécies $(8,57 \%)$, Annonaceae e Malvaceae com quatro espécies cada $(5,71 \%)$ e Bignoniaceae, Meliaceae, Myrtaceae e Sapindaceae com três espécies cada $(4,28 \%)$. O restante das famílias $(19 ; 27,14 \%)$ apresentou apenas uma espécie.

O índice de Shannon (H') calculado foi de 3,44 (nats/ind.) e a equabilidade (J'), de 0,81. Esse valor de diversidade pode ser considerado alto se comparado com o de outras florestas com condições similares de saturação hídrica do solo e seletividade de espécies, como as florestas de brejo (TORRES et al., 1994; IVANAUSKAS et al., 1997; PASCHOAL et al., 1998; MARQUES, 2003) e próximo ao obtido em vegetação de cerrado e florestas de galeria do Brasil central (FELFILI et al., 1993; FELFILI e SILVAJÚNIOR, 1993; FELFILI, 1994; FELFILI et al., 2002; ANDRADE et al., 2002). A maior diversidade na floresta inundável do presente estudo, em comparação com florestas de brejo, deve ser resultado do período livre de inundação a que a primeira está submetida anualmente, o que possibilita melhor drenagem do solo na estação seca. Na floresta de brejo, o solo apresenta permanente excesso de água, tornando-se ainda mais seletivo às espécies arbóreas (TORRES et al., 1994; IVANAUSKAS et al., 1997).

As espécies que apresentaram maior número de indivíduos foram aquelas que tiveram maior valor de cobertura (Quadro 2). Hirtella racemosa, a espécie com VC mais alto $(23,02)$, exibiu também a maior densidade e dominância relativa. As demais espécies com maior VC destacaram-se por apresentar maior densidade, como Qualea multiflora, Byrsonima intermedia, Curatella americana, Xylopia aromatica, Eschweilera ovata, Tapirira obtusa, Zanthoxylum riedelianum e Copaifera langsdorffii. Essas espécies ocorrem tanto em áreas de Cerrado, quanto como de floresta (FREITAS e OLIVEIRA, 2002; ANDRADE et al., 2002).

Tabela 2 - Espécies amostradas no fragmento de floresta inundável e seus parâmetros fitossociológicos: $\mathrm{n}=$ número de indivíduos; DA = densidade absoluta, DoA = aominância absoluta, DR = densidade relativa; DoR = dominância relativa e $\mathrm{VC}=$ valor de cobertura

Table 2 - Sampled species in the flooded forest fragment and their phytosociological parameters: $n=$ number of individuals; $D A=$ absolute density, $D o A=$ absolute dominance,$D R=$ relative density $;$ Do $R=$ relative dominance $; V C=$ cover value

\begin{tabular}{|c|c|c|c|c|c|c|}
\hline Espécie & Ind. & DA & DoA & DR & DoR & $\mathrm{VC}$ \\
\hline Hirtella racemosa Lam. & 88 & 77,2 & 1,5656 & 10,90 & 12,11 & 23,02 \\
\hline Qualea multiflora Mart. & 71 & 62,3 & 0,7850 & 8,80 & 6,07 & 14,87 \\
\hline Cecropia pachystachya Trécul & 70 & 61,4 & 0,6693 & 8,67 & 5,18 & 13,85 \\
\hline Curatella americana $\mathrm{L}$. & 45 & 39,5 & 0,8950 & 5,58 & 6,92 & 12,05 \\
\hline Tapirira obtusa (Benth.) D.J. Mitch. & 37 & 32,5 & 0,8951 & 4,58 & 6,93 & 11,51 \\
\hline Eschweilera ovata (Cambess.) Miers & 41 & 36,0 & 0,8099 & 5,08 & 6,27 & 11,35 \\
\hline Byrsonima intermedia A. Juss. & 60 & 52,6 & 0,4685 & 7,43 & 3,62 & 11,06 \\
\hline Copaifera langsdorffii Desf. & 24 & 21,1 & 0,6393 & 2,97 & 4,95 & 7,92 \\
\hline Xylopia aromatica (Lam.) Mart. & 29 & 25,4 & 0,5307 & 3,59 & 4,11 & 7,70 \\
\hline Zanthoxylum riedelianum Engl. & 33 & 28,9 & 0,4198 & 4,09 & 3,25 & 7,34 \\
\hline Virola sebifera Aubl. & 18 & 15,8 & 0,3925 & 2,23 & 3,04 & 5,27 \\
\hline Emmotum nitens (Benth.) Miers & 13 & 11,4 & 0,4385 & 1,61 & 3,39 & 5,00 \\
\hline Qualea grandiflora Mart. & 22 & 19,3 & 0,2351 & 2,73 & 1,82 & 4,54 \\
\hline Maprounea guianensis Aubl. & 18 & 15,8 & 0,2010 & 2,23 & 1,55 & 3,79 \\
\hline Tabebuia serratifolia (Vahl) G. Nicholson & 4 & 3,5 & 0,3984 & 0,50 & 3,08 & 3,58 \\
\hline Syagrus cocoides Mart. & 19 & 16,7 & 0,1112 & 2,35 & 0,86 & 3,22 \\
\hline Calophyllum brasiliense Cambess. & 13 & 11,4 & 0,1467 & 1,61 & 1,13 & 2,75 \\
\hline Aspidosperma subincanum Mart. & 9 & 7,9 & 0,1860 & 1,12 & 1,44 & 2,55 \\
\hline
\end{tabular}

R. Árvore, Viçosa-MG, v.30, n.5, p.829-836, 2006 
Quadro 2 - Cont.

Table 2-Cont.

\begin{tabular}{|c|c|c|c|c|c|c|}
\hline Espécie & Ind. & $\mathrm{DA}$ & DoA & DR & DoR & $\mathrm{VC}$ \\
\hline$\overline{\text { Aegiphila lhotskiana Cham. }}$ & 2 & 1,8 & 0,2950 & 0,25 & 2,28 & 2,53 \\
\hline Protium heptaphyllum (Aubl.) Marchand & 8 & 7,0 & 0,1582 & 0,99 & 1,22 & 2,22 \\
\hline Astrocaryum vulgare Mart. & 9 & 7,9 & 0,1192 & 1,12 & 0,92 & 2,04 \\
\hline Byrsonima indorum S. Moore & 6 & 5,3 & 0,1641 & 0,74 & 1,27 & 2,01 \\
\hline Nectandra gardneri Meisn. & 7 & 6,1 & 0,0977 & 0,87 & 0,76 & 1,62 \\
\hline Machaerium opacum Vogel & 4 & 3,5 & 0,1330 & 0,50 & 1,03 & 1,52 \\
\hline Myrcia fallax (Rich.) DC. & 4 & 3,5 & 0,1308 & 0,50 & 1,01 & 1,51 \\
\hline Physocalymma scaberrimum Pohl & 7 & 6,1 & 0,0823 & 0,87 & 0,64 & 1,50 \\
\hline Ceiba sp. & 2 & 1,8 & 0,1599 & 0,25 & 1,24 & 1,48 \\
\hline Protium grandifolium Engl. & 5 & 4,4 & 0,1069 & 0,62 & 0,83 & 1,45 \\
\hline Plathymenia reticulata Benth. & 7 & 6,1 & 0,0736 & 0,87 & 0,57 & 1,44 \\
\hline Duguetia megalocarpa Maas & 7 & 6,1 & 0,0706 & 0,87 & 0,55 & 1,41 \\
\hline Hymenaea courbaril $\mathrm{L}$. & 3 & 2,6 & 0,1301 & 0,37 & 1,01 & 1,38 \\
\hline Lafoensia pacari A. St.-Hil. & 7 & 6,1 & 0,0398 & 0,87 & 0,31 & 1,18 \\
\hline Diospyros sericea A. DC. & 5 & 4,4 & 0,0571 & 0,62 & 0,44 & 1,06 \\
\hline Heisteria laxiflora Engl. & 5 & 4,4 & 0,0498 & 0,62 & 0,38 & 1,00 \\
\hline Matayba guianensis Aubl. & 4 & 3,5 & 0,0614 & 0,50 & 0,47 & 0,97 \\
\hline Callisthene fasciculata Mart. & 4 & 3,5 & 0,0585 & 0,50 & 0,45 & 0,95 \\
\hline Roupala montana Aubl. & 5 & 4,4 & 0,0404 & 0,62 & 0,31 & 0,93 \\
\hline Cordia alliodora (Ruiz \& Pav.) Oken & 6 & 5,3 & 0,0240 & 0,74 & 0,19 & 0,93 \\
\hline Luehea divaricata Mart. & 3 & 2,6 & 0,0655 & 0,37 & 0,51 & 0,88 \\
\hline Trichilia micrantha Benth. & 2 & 1,8 & 0,0678 & 0,25 & 0,52 & 0,77 \\
\hline Sclerolobium aureum (Tu1.) Baill. & 3 & 2,6 & 0,0516 & 0,37 & 0,40 & 0,77 \\
\hline Guatteria pubens (Mart.) R.E.Fr. det. J.E. Simonis & 4 & 3,5 & 0,0335 & 0,50 & 0,26 & 0,75 \\
\hline Jacaranda brasiliana (Lam.) Pers. & 4 & 3,5 & 0,0251 & 0,50 & 0,19 & 0,69 \\
\hline Allophylus eduli s(A. St.-Hil., Cambess. \& A. Juss.)Radlk & 2 & 1,8 & 0,0485 & 0,25 & 0,38 & 0,62 \\
\hline Qualea dichotoma (Mart.) Warm. & 3 & 2,6 & 0,0282 & 0,37 & 0,22 & 0,59 \\
\hline Tabebuia aurea (Silva Manso)Benth. \& Hook. f. ex S. Moore & 3 & 2,6 & 0,0278 & 0,37 & 0,21 & 0,59 \\
\hline Matayba elaeagnoides Radlk. & 3 & 2,6 & 0,0220 & 0,37 & 0,17 & 0,54 \\
\hline Vochysia rufa Mart. & 3 & 2,6 & 0,0216 & 0,37 & 0,17 & 0,54 \\
\hline Trichilia lepidota Mart. & 1 & 0,9 & 0,0449 & 0,12 & 0,35 & 0,47 \\
\hline Swartzia apetala Raddi & 1 & 0,9 & 0,0427 & 0,12 & 0,33 & 0,45 \\
\hline Xylopia sericea A. St.-Hil. & 2 & 1,8 & 0,0205 & 0,25 & 0,16 & 0,41 \\
\hline Zanthoxylum rhoifolium Lam. & 2 & 1,8 & 0,0187 & 0,25 & 0,14 & 0,39 \\
\hline Ocotea aciphylla (Nees) Mez & 2 & 1,8 & 0,0147 & 0,25 & 0,11 & 0,36 \\
\hline Siparuna glycycarpa (Ducke)S. S. Renner \& Hausner & 2 & 1,8 & 0,0131 & 0,25 & 0,10 & 0,35 \\
\hline Erythroxylum anguifugum Mart. & 2 & 1,8 & 0,0130 & 0,25 & 0,10 & 0,35 \\
\hline Dipteryx alata Vogel & 1 & 0,9 & 0,0230 & 0,12 & 0,18 & 0,30 \\
\hline Hirtella glandulosa Spreng. & 2 & 1,8 & 0,0072 & 0,25 & 0,06 & 0,30 \\
\hline Eugenia florida DC. & 2 & 1,8 & 0,0066 & 0,25 & 0,05 & 0,30 \\
\hline Trichilia hirta $\mathrm{L}$. & 1 & 0,9 & 0,0213 & 0,12 & 0,16 & 0,29 \\
\hline Cannabaceae sp. & 1 & 0,9 & 0,0201 & 0,12 & 0,16 & 0,28 \\
\hline Acosmium dasycarpum (Vogel) Yakovlev & 2 & 1,8 & 0,0041 & 0,25 & 0,03 & 0,28 \\
\hline Calyptranthes concinna DC. & 1 & 0,9 & 0,0168 & 0,12 & 0,13 & 0,25 \\
\hline Apeiba tibourbou Aubl. & 1 & 0,9 & 0,0148 & 0,12 & 0,11 & 0,24 \\
\hline Ouratea castaneifolia (DC.) Engl. & 1 & 0,9 & 0,0114 & 0,12 & 0,09 & 0,21 \\
\hline Myracrodruon urundeuva Allemao & 1 & 0,9 & 0,0059 & 0,12 & 0,05 & 0,17 \\
\hline Luehea grandiflora Mart. & 1 & 0,9 & 0,0047 & 0,12 & 0,04 & 0,16 \\
\hline Vochysia divergens Poh1 & 1 & 0,9 & 0,0047 & 0,12 & 0,04 & 0,16 \\
\hline Guettarda virbunoides Cham. \& Schltdl. & 1 & 0,9 & 0,0042 & 0,12 & 0,03 & 0,16 \\
\hline Senna spectabilis (DC.) H.S. Irwin \& Barneby & 1 & 0,9 & 0,0034 & 0,12 & 0,03 & 0,15 \\
\hline Caryocar villosum (Aub1.) Pers. & 1 & 0,9 & 0,0027 & 0,12 & 0,02 & 0,14 \\
\hline
\end{tabular}


Qualea multiflora, Byrsonima intermedia, Curatella americana e Xylopia aromatica já haviam sido descritas como ocorrentes em áreas do Cerrado (FREITAS e OLIVEIRA, 2002; ANDRADE et al., 2002; MARQUES et al., 2003) e certamente estão estabelecidas nos fragmentos de florestas inundáveis da área do presente estudo, devido ao período sem encharcamento do solo. A abundância de Cecropia pachystachya e Calophyllum brasiliense, típicas de solos encharcados das florestas ribeirinhas e de brejo (TORRES et al., 1994; IVANAUSKAS et al., 1997; TONIATO et al., 1998; RODRIGUES e NAVE, 2000, MARQUES et al., 2003), explicar-se-ia pelo período de encharcamento sazonal na floresta inundável e pela pouca profundidade do lençol freático na estação seca.

Observou-se, também, que algumas espécies com baixa densidade como Aegiphila lhotskiana (dois indivíduos) e Tabebuia serratifolia (quatro indivíduos) apresentam altos valores de dominância, devido ao fato de seus indivíduos apresentarem elevada circunferência na altura do peito (CAP).
Acredita-se que Swartzia apetala, Trichilia lepidota, Trichilia hirta, Protium grandifolium e Machaerium opacum, representadas por um único indivíduo na área estudada, possam ser raras por se tratarem de espécies de estádio avançado na sucessão, ou em razão das alterações antrópicas no ambiente.

As espécies com maior VC foram individualizadas para representar suas distribuições de indivíduos em classes de diâmetro (Figura 1bf) Hirtella racemosa, Qualea multiflora, Cecropia pachystachya, Curatella americana e Tapirira obtusa.

A distribuição do número de indivíduos por classe diamétrica para a comunidade como um todo apresentou a predominância nas três menores classes de DAP $(82,77 \%)$, gerando um gráfico com a configuração de "J invertido" (Figura 1a). Esse padrão representa grande quantidade de indivíduos jovens no fragmento.

As espécies Hirtella racemosa, Qualea multiflora e Cecropia pachystachya (Figuras 1b-d) destacam-se na comunidade por apresentarem as maiores densidades (88, 71 e 70 indivíduos, respectivamente) em relação às demais.
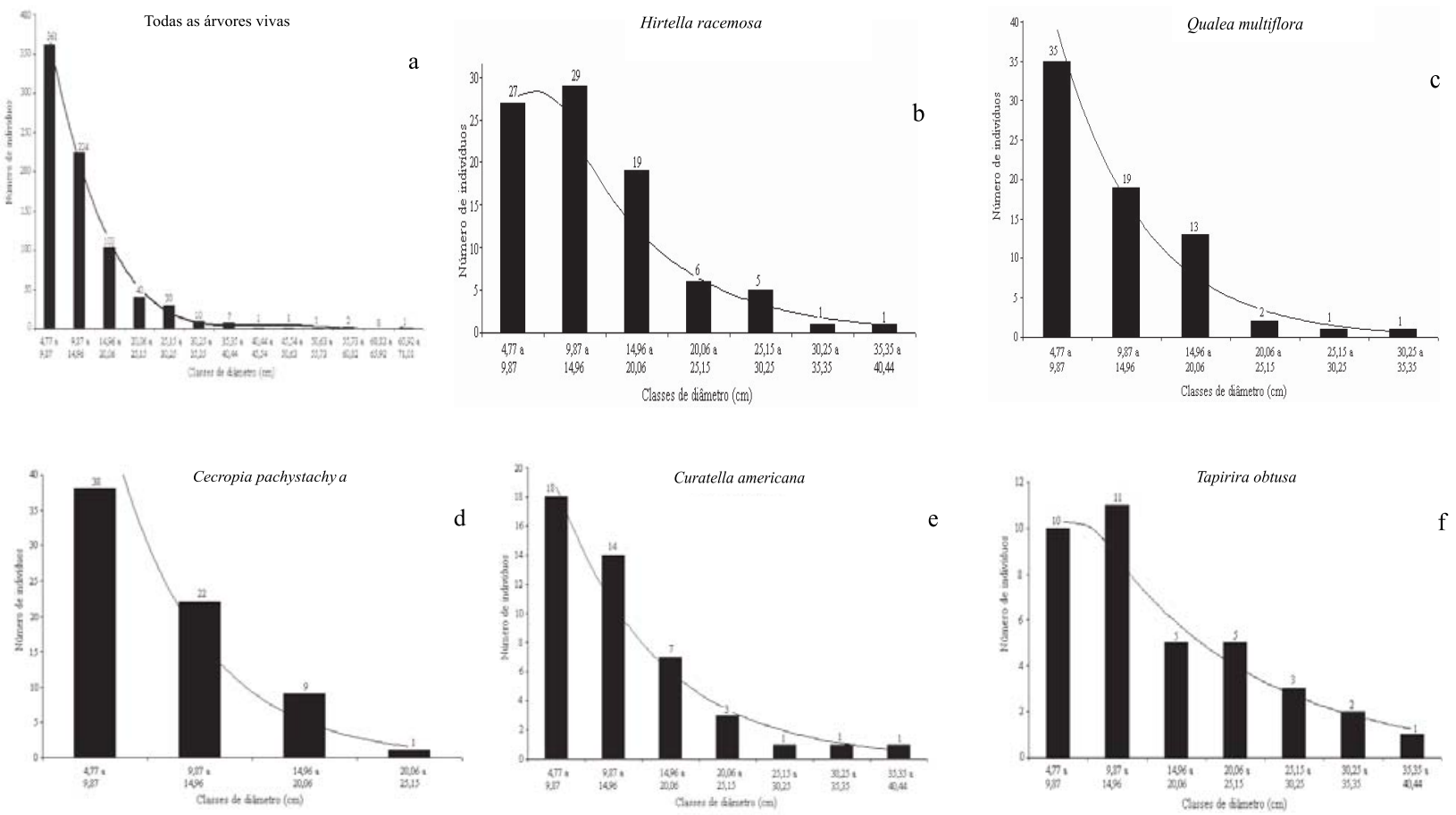

Figura 1 - Distribuição em classes de diâmetro do total de indivíduos amostrados (a) e dos indivíduos das espécies de maior VC (b-f).

Figure 1 -Distribution in Diameter classes, for all individuals sampled and individuals from species of larger VC.

R. Árvore, Viçosa-MG, v.30, n.5, p.829-836, 2006 
Hirtella racemosa (Figura 1b) e Tapirira obtusa (Figura 1f) diferem das demais espécies em relação à distribuição diamétrica por apresentarem baixo recrutamento, provavelmente devido ao avanço sucessional que está em curso. Esse fato pode indicar que não está havendo regeneração satisfatória nas populações destas espécies. No entanto, Qualea multiflora, Cecropia pachystachya e Curatella americana (Figura 1c-e) apresentaram distribuição diamétrica balanceada dos seus indivíduos, na conformação de um " J" invertido, mostrando que suas populações estão em bom estado de conservação e aparentemente sem problemas de regeneração.

A importância da conservação dessas florestas de ipucas torna-se de fundamental importância, dado o pouco conhecimento acumulado sobre elas. Essa diversidade certamente está relacionada com a atuação e conservação da fauna nas áreas de orizicultura, ou seja, as florestas inundáveis funcionam como elos na paisagem antropizada, fazendo a ligação entre fragmentos de Cerrado, de galeria e Floresta Amazônica. Portanto, proteger a diversidade das áreas onde ocorrem as ipucas pode ser considerado um fator relevante na região, uma vez que atividades como a expansão agropecuária vêm avançando em desconformidade com o conhecimento científico desses fragmentos naturais.

\section{RECOMENDAÇÕES}

Neste contexto, a partir do conhecimento dos aspectos essenciais à sustentabilidade das florestas inundáveis como composição florística, riqueza de espécies e organização estrutural, obtido neste estudo, é possível discutir medidas que visam desencadear na comunidade local atividades de educação ambiental com relação à importância de se conservar a cobertura vegetal desses fragmentos em conformidade com desenvolvimento agropecuário de forma consciente e subsidiar, através desse estudo, as políticas públicas do Estado do Tocantins para eficientes tomadas de decisões, visando à conservação e restauração dessas florestas.

\section{REFERÊNCIAS BIBLIOGRÁFICAS}

ANDRADE, L.A.Z.; FELFILI, J.M.; VIOLATTI, L. Fitossociologia de uma área de cerrado denso na RECOR-IBGE, Brasília-DF. Acta Botanica Brasilica, v. 16, n. 2, p. 225-240. 2002.
ANGIOSPERM PHYLOGENY GROUP-APG. An update of the Angiosperm Phylogeny Group classification for the orders and families of flowering plants: APG II. Botanical Journal of the Linnean Society, v. 141, p. 399-436. 2003.

BORÉM, R.A.T.; OLIVEIRA-FILHO, A.T.

Fitossociologia do estrato arbóreo em uma toposseqüência alterada de mata atlântica, no município de Silva Jardim-RJ, Brasil. Revista Árvore, v. 26, n. 6, p. 727-742, 2002.

COUTINHO, L.M. O Cerrado e a ecologia do fogo. Ciência Hoje, v. 12, n. 68, p. 23-30. 1990.

EITEN, G. Vegetation near Santa Teresinha, NE Mato Grosso. Acta Amazônica v. 15, n. 3-4, p. 275-301, 1985.

EITEN, G. Vegetação dos cerrados. In: PINTO, M.N. Cerrado: caracterização, ocupação e perspectivas. 2. ed. Brasília: UNB, SEMATEC. 1994. p. 9-65.

EMPRESA BRASILEIRA DE PESQUISA AGROPECUÁRIA - EMBRAPA. Manual de métodos de análise de solo. Rio de Janeiro: EMBRAPA - SNLCS, 1979.

EMPRESA BRASILEIRA DE PESQUISA AGROPECUÁRIA - EMBRAPA. Manual de métodos de análise de solo. Rio de Janeiro: EMBRAPA - SNLCS, 1999.

FACHIM, E.; GUARIM, V.L.M.S. Conservação da biodiversidade: espécies da flora de Mato Grosso. Acta Botanica Brasilica, v. 9, n. 2, p. 281-287, 1995.

FELFILI, J.M.; SILVA JÚNIOR, M.C. Distribuição dos diâmetros numa faixa de Cerrado na fazenda água Limpa (FAL) em Brasília-DF. Acta Botanica Brasilica, v. 2, n. 1-2, p. 85-104, 1988.

FELFILI, J.M. et al. Análise comparativa da florística e fitossociologia da vegetação arbórea do Cerrado sensu stricto na Chapada Pratinha, DF-Brasil. Acta Botonica Brasilica, v. 6, n. 2, p. 27-46, 1993.

FELFILI, J. M.; SILVA JÚNIOR, M. C. A comparative study of cerrado (sensu stricto) vegetation in Central Brazil. Journal Tropical of Ecology, v. 9, p. 277-289, 1993.

R. Árvore, Viçosa-MG, v.30, n.5, p.829-836, 2006 
FELFILI, J.M. Floristic composition and phytosociology of the gallery forest alongside the Gama stream in Brasília, DF, Brazil. Revista

Brasileira de Botânica, v. 17, n. 1, p. 1-11, 1994.

FELFILI, J.M. et al. Composição florística e fitossociologia do Cerrado sentido restrito no município de Água Boa-MT. Acta Botanica Brasilica, v. 16, n. 1, p. 103-112, 2002.

FIEDLER, N.C. et al. Efeito de incêndios florestais na estrutura e composição florística de uma área de cerrado sensu stricto na fazenda Água Limpa-DF. Revista Árvore, v. 28, n. 1, p. 129-138, 2004.

FORERO, E. Los jardines botánicos y la conservación de la natureza. Acta Botanica Brasilica, v. 3, n. 2, p. 315-322, 1989.

FREITAS, C.V.; OLIVEIRA, P.E. Biologia reprodutiva de Copaifera langsdorffii Desf. (Leguminosae, Caesalpinioideae). Revista Brasileira de Botânica, v.25, n.3, p.311-321. 2002.

HARPER, J.L. Population biology of plants. London: Academic Press, 1977.

IVANAUSKAS, N.M., RODRIGUES, R.R. \& NAVE, A.G. Aspectos ecológicos de um trecho de Florestas de Brejo em Itatinga, SP: florística, fitossociologia e seletividade de espécies. Revista Brasileira de Botânica, v. 20, n. 2, p. 139-153, 1997.

MARQUES, M.C.M.; SILVA, S. M.; SALINO, A. Florística e estrutura do componente arbustivoarbóreo de uma floresta higrófila da bacia do rio Jacaré-Pepira, SP, Brasil. Acta Botanica Brasilica, v. 17, n. 4, p. 495-506, 2003.

MARTINS, I. C. M. et al. Diagnóstico ambiental no contexto da paisagem de fragmentos florestais naturais "ipucas" - no Município de Lagoa da Confusão, Tocantins. Revista Árvore, v. 26, n. 3, p. 299-309, 2002.

MITTERMEIR, R.A. et al. O país da megadiversidade. Ciência Hoje, v. 14, n. 81, p. 20-27, 1992.

MUELLER DOMBOIS, D.Y.; ELLENBERG, M. Aims and methods in vegetation ecology. New York: Willey and Sons, 1974. 547 p.

R. Árvore, Viçosa-MG, v.30, n.5, p.829-836, 2006
OLIVEIRA-FILHO, A. T.; RATTER, J. A. A study of the origin of central Brazilian forests by the analysis of plant species distribution patterns. Edinburg Journal of Botany, v. 52, n. 2, p. 141-194, 1995.

PASCHOAL, M.E.S.; CAVASSAN, O. A flora arbórea da mata de brejo do ribeirão Pelintra, Agudos, SP. Naturalia, v. 24, p. 172-191, 1999.

PIELOU, E.C. The interpretation of ecological data. New York: John Wiley \& Sons, 1975. 263p.

RATTER, J. A.; RIBEIRO, J. F.; BRIDGEWATER, S. The Brazilian cerrado vegetation and threats to its biodiversity. Annals of Botany, v. 80, n. 3, p. 223-230, 1997.

RODRIGUES, R. R.; NAVE, A. A. Heterogeneidade florística das Matas Ciliares. In RODRIGUES, R. R. e LEITÃO-FILHO, H. F. (Eds.). Matas ciliares: conservação e recuperação. São Paulo: EDUSP/FAPESP, 2000. p. 45-71.

SAUNDERS, D. A.; HOBBS, R. J.; MARGULES, C. R. Biological consequences of fragmentation: A review. Conservation Biology, v. 5, p. 18-32. 1991.

SEPLAN/TO. Atlas do Tocantins: Subsídios ao planejamento da gestão territorial. 2. ed. Palmas: Secretaria do Planejamento e Meio Ambiente, Diretoria de Zoneamento EcológicoEconômico-DZEE. 2002. 49 p.

SILVA, L.O. et al. Levantamento florístico e fitossociológico em duas áreas de Cerrado sensu stricto no Parque Estadual da Serra de Caldas Novas, Goiás. Acta Botanica Brasilica, v. 16, n. 1, p. 43-53, 2002.

SHEPHERD, G. J. Fitopac 1: manual do usuário. Campinas, Universidade de Campinas, 1996.

TONIATO, M. T. Z.; LEITÃO-FILHO, H. F.; RODRIGUES, R. R. Fitossociologia de um remanescente de Floresta higrófila (Mata de brejo) em Campinas, SP. Revista Brasileira de Botânica, v. 2, n. 21, p. 197-210, 1998.

TORRES, R.B.; MATHES, L.A.F.; RODRIGUES, R.R. Florística e estrutura do componente arbóreo de mata de brejo em Campinas, SP. Revista Brasileira de Botânica, v. 17. n. 2, p. 189-194, 1994. 